\title{
La condena de la avaricia en Purgatorio a partir del tratado Monarquía. ${ }^{1}$
}

\section{Greed condemnation in Purgatorio from Monarchia treatise.}

DOI: $10.32870 /$ sincronia.axxv.n79.16a21

\section{Ricardo Manuel Leyva Gama}

Universidad Anáhuac México Sur (MÉXICO)

CE: leyva ricardo@outlook.com / ID ORCID: 0000-0002-4791-688X

Esta obra está bajo una Licencia Creative Commons Atribución-NoComercial 4.0 Internacional

Recibido: 08/09/2020

Revisado: 03/10/2020

Aprobado: $19 / 11 / 2020$

\section{RESUMEN}

La avaricia es el pecado más condenado por Dante Alighieri en su Comedia. Para comprender la dimensión de esta condena, en este artículo se opta por una lectura dualista del Purgatorio, i.e., una que permite considerar lo que entiende la razón de la virtud y el vicio humano y lo que hay que entender por la fe cristiana. Para lograr esto, se revisan algunas ideas teológicas y políticas de Dante en su tratado Monarquía.

Palabras Clave: Avaricia. Dante Alighieri. Purgatorio. Monarquía.

\section{ABSTRACT}

Greed is the most condemned sin by Dante Alighieri in his Comedy. To understand this condemnation, in this paper, it is opted for a dualist lecture of Purgatory, i.e., one that allows us to consider both what reason understands human virtue and vice and what one must accept by the Christian faith. To achieve this, some of Dante's theological and political ideas are recovered from his treatise Monarchia.

Keywords: Greed. Dante Alighieri. Purgatory. Monarchia.

\footnotetext{
${ }^{1}$ Este artículo ha sido elaborado en el marco del programa de "Becas Doctorales" de la Facultad de Humanidades, Filosofía y Letras, del Doctorado en Filosofía de la Universidad Anáhuac, Campus Sur, coordinado por el Dr. Rafael García Pavón a quien agradezco su valiosa asesoría y permanente acompañamiento.
} 
De los pecados incontinentes, i.e., cuya naturaleza radica en el exceso o el defecto de una acción, la avaricia es el pecado más condenado por Dante en su Comedia. Cuantitativamente, el círculo infernal de la avaricia es el más poblado (Infierno, VII, 20); cualitativamente, por voz de Adriano V, sabemos que la pena de los avaros es la más severa en el purgatorio (Purgatorio, XIX, 117). ${ }^{2}$ Al inicio del poema, el mismo pecado aparece representado por una loba, junto a la lujuria y la soberbia, simbolizadas por una pantera y un león, respectivamente (Inferno, I, 31-54), siendo éstos pecados los que predominan en el mundo, y los que causaron muchas de las amarguras de Dante. En este trabajo, quiero examinar cómo se da la condena a la avaricia. Para esto, primero expondré cómo clasifica Dante a los pecados; seguido, revisaré algunas de sus afirmaciones en el tratado Monarquía; posteriormente, vincularé lo revisado con algunas partes de los cantos XVI al XX del Purgatorio $^{3}$ y concluiré con algunas reflexiones breves.

Por el canto XI del Infierno, sabemos que la estructura de éste se basa en la filosofía moral de Aristóteles en la Ética a Nicómaco. A grandes rasgos, el bajo infierno - del segundo al quinto círculo - contiene a los pecadores incontinentes, i.e., que vivieron en excesos o defectos en sus actos; por otra parte, el alto infierno - del sexto al noveno círculo - contiene a los pecadores que cometieron algún mal absoluto, i.e., que no acepta un término medio o que implicó el uso de la razón para hacer daño al prójimo. En el canto XVII del Purgatorio nos encontramos con una explicación similar del orden del purgatorio. Por medio de Virgilio tenemos noticia de que todo, tanto el Creador como sus creaturas, irradia amor. La forma en la cual se dirija éste determina cuándo se vive virtuosamente o en algún vicio. Debido a que el amor busca la felicidad de quien lo porta, el odio a uno mismo es imposible; y debido a que todo tiene a Dios por causa, nadie puede odiarlo a Él. De esta forma, el odio sólo es posible al prójimo y a las cosas creadas. Sentados estos principios, Virgilio le explica a Dante que en el purgatorio hay una clasificación triple: el amor deforme, que odia al prójimo y a su bienestar para elevarse a uno mismo, el cual corresponde a la

\footnotetext{
${ }^{2}$ Que la avaricia es además el pecado de mayor alcance se reitera en Purgatorio, XIX, 11-12.

${ }^{3}$ Aunque en el canto XXI del Purgatorio Dante sigue en la cornisa de los avaros, éste va más dirigido a los pródigos y al encuentro de Dante y Virgilio con Estacio.
} 
soberbia, la ira y la envidia; el amor deficiente, que corresponde a la pereza; y el amor excesivo, que corresponde a la avaricia, la gula y la lujuria.

Prima facie, el purgatorio puede parecer como un intermedio entre el infierno y el paraíso. Quien no fue un pecador cuyo pecado le haya ganado el infierno, pero tampoco un alma cuyas obras le merecieran el paraíso, tiene que pasar por el purgatorio para hacerse puro y subir a la perfección de los cielos. Aún más, el hecho de que haya pecados que se presentan en ambos lugares - como la gula, la lujuria, la avaricia y la ira - da un mayor sustento a esta idea. Aun con todo, en la literatura del último siglo se ha promovido más bien una lectura dualista del Purgatorio. Mientras el infierno está regido por lo que la sola razón puede comprender del orden moral en el mundo, el purgatorio es un camino del alma desde el amor a lo terrenal hacia el amor de las cosas divinas. Así pues, la estructura de éste se basa más bien en el recto amor hacia Dios, y, por tanto, su estructura es más bien teológica (Corbett, 2014). A favor de esto, basta con observar que el orden moral que rige a sus cornisas son los siete pecados capitales y que en la puerta del purgatorio se aparece el proceso de penitencia representado por tres escalones que simbolizan la consciencia, el pecado y la sangre de Cristo respectivamente (Purgatorio, IX, 94-102). Esta lectura dualista nos sugiere buscar no sólo las razones filosóficas o racionales para condenar la avaricia - como un exceso - sino también los motivos teológicos para hacerlo.

A partir de este tipo de lectura uno puede recuperar algunas ideas teológico-políticas de Dante en su tratado Monarquía. En este breve texto, Dante se suma a una discusión propia de su tiempo: ¿el poder temporal es independiente del poder espiritual? Más allá del ámbito teológicofilosófico, los bandos políticos se dividían entonces entre los güelfos - en su mayoría nobles -, quienes creían que ambos poderes deben ir juntos, y los gibelinos - en su mayoría nuevos ricos (burgueses) -, quienes creían que los poderes son independientes. Mientras Dante vivió en Florencia, él fue güelfo por la tradición en su familia, aun cuando pertenecía a la facción blanca de estos, la cual defendía la independencia política de Florencia con respecto al papa; sin embargo, tras sus experiencias en el destierro, Dante se volcó más hacia el lado de los gibelinos (Díaz Mendieta, 2013, 311). 
Pudieron existir varios motivos por los cuales Dante se decidió a escribir su tratado Monarquía. Algunos estudiosos datan el inicio de redacción del tratado en 1310 con el descenso de Enrique VII a Italia y la interrupción en 1314 al morir éste (Gutiérrez, 1994, pp. 694s). Por la carta que Dante le dirige a este emperador, es plausible suponer que un motivo era su deseo de regresar a Florencia, pues Enrique VII no era precisamente un rey muy exitoso en sus empresas (Díaz, 2013, p. 313). Cualquiera que fuese el motor de la redacción, en su tratado Dante se decidió a dirigir una crítica abierta al pontificado romano por sus relaciones próximas con el poder temporal, más específicamente con reyes franceses.

El tratado consta de tres libros, cada uno de los cuales tiene un argumento central. El primero defiende a la monarquía como la forma de gobierno que sirve mejor a la felicidad de los hombres. Entre otras, las tesis centrales de Dante en este texto son:

(a) La paz es el mejor medio para que los hombres consigan su felicidad. $(I, 4)$

(b) El mejor gobierno es único y unifica a los pueblos. $(1,5)$

(c) La monarquía ordena todo a un mismo fin, que es la paz. $(I, 7)$

(d) El monarca único es el más justo de todos porque no apetece nada. $(I, 11)$

(e) El monarca quiere que cada quien viva para sí mismo, i.e., no somete. $(I, 12)$

(f) La monarquía de Augusto fue un tiempo feliz y pleno. $(\mathrm{I}, 16)$

Algunas de estas tesis pueden parecer ingenuas. Al respecto, Ángel Crespo ha señalado que a Dante lo mueve más una razón poética que una científica, por lo cual no es raro encontrarse muchas peticiones de principio en su argumentación (1999, pp. 82s); sin embargo, para nuestro propósito nos conviene tener presentes estas ideas de Dante.

El segundo libro se dedica a demostrar, por fuentes históricas y poéticas, que el imperio romano fue legítimo en tanto que fue una voluntad de Dios. Aquí no detallaremos esto.

Finalmente, el tercer libro contesta a la cuestión: ¿la autoridad del monarca depende de Dios o de algún ministro o vicario de Dios? Como lo apunta Dante en su apertura, su respuesta es 
desfavorable para el pontífice romano y beneficiosa para el príncipe. Dante creía que la autoridad del monarca deriva directamente de Dios y no de alguno de sus vicarios o ministros.

Algunos argumentos de Dante a favor de la separación son los siguientes. Primero, niega una lectura del génesis en la cual se dice que el Sol y la Luna representan los dos poderes, diciendo que Dios crearía antes el remedio que el problema, pues éstos anteceden a la creación del hombre que aún no ha pecado y no necesita una guía en un estado así; aún más esta lectura más bien sugiere que ambos poderes son distintos e independientes, incluso cuando el auxilio de uno al otro puede iluminar mejor al mundo (III, 4). Otro pasaje que Dante niega es una respuesta directa a una lectura de Bonifacio VIII, a quien Dante consideró la causa de su destierro al enviar a Carlos de Valois a Florencia. Bonifacio VIII defendió la unión de los poderes basado en un pasaje del Evangelio de Lucas en el que Pedro tiene dos espadas, lo cual significa que Pedro, su vicario, tiene ambos poderes. Dante resignifica el pasaje diciendo que Jesús precavió a todos sus apóstoles del peligro que vendría en su captura, por lo cual les pide a todos, no sólo a Pedro, que tengan una espada (III, 9).

Finalmente, Dante rechazaba uno de los fundamentos de la autoridad política de la iglesia: la donación de Constantino (III, 10). En aquel entonces, se creía que Constantino había donado la mitad de su imperio al papa Silvestre I, quien le curara de una enfermedad; con el imperio occidental, el papa se quedaría entonces con Roma, la capital del imperio. Un par de siglos después de la muerte de Dante el documento que validaba el hecho se declaró como falso; sin embargo, por un largo tiempo se creyó que esto daba una figura política al sucesor de Pedro (Barceló, 2003, pp. 55-57).

Como tal Dante consideraba que la intención de Constantino era buena, pero que su acción destruyó al mundo (Paraíso, XX, 58-60), pues había enriquecido al primer padre (Infierno, XIX, 115117). En contra de este principio jurídico, Dante veía un problema bilateral. Ni Constantino podía donar el imperio ni la iglesia podía recibirlo. Sobre lo primero, Dante tomaba una afirmación de tercio excluso. Constantino era o no emperador al hacer la donación. Si era emperador, no podía donar su imperio porque el emperador trae unidad y no separa su dominio. Si no era emperador, no 
podía hacer la donación legalmente. En cuando a lo segundo, Dante indica que, aún si Constantino hubiera podido realizar la donación, la iglesia no podía recibirlo porque en el Evangelio según San Mateo dice «no poseáis oro ni plata» (10, 9, versión Reina Valera), y que su relajación es muy limitada (Lucas, 9, 3). No se predicó entonces la pobreza evangélica.

Ahora bien, algunas de las ideas que hemos revisado del tratado Monarquía se hallan al fondo del Purgatorio y es muy posible que ambas obras fueran redactadas a la par (Crespo, 1999, p.87), por lo cual también se complementan.

Como tal, encontraremos a los avaros hasta el canto XIX del Purgatorio, pero desde el canto XVI podemos apuntar algunos paralelismos que nos aclaran más la causa del castigo. Así pues, comenzaremos con la conversación con Marco Lombardi en la cornisa de los iracundos. Cabe apuntar que el nombre genérico y la falta de información del personaje ha llevado a comentaristas a creer que Dante puso sus propios pensamientos en otro personaje a parte de él mismo (Chiclana, 2002, p. 305; Echeverría, 2013, p. 304), por lo cual, esto sería una especie de monólogo de Dante. Marco Lombardi le dice a Dante que en el mundo ya nadie tiende el arco de la virtud, y su explicación es la siguiente:

el alma simplecilla, sin pericia, pero, movida por feliz autor, se inclina a cuanto piensa ser delicia.

En leve bien primero halla sabor, pero se engaña y, por lograrlo, corre si rienda o freno no tuercen su amor La buena ley la frena y la socorre, que un rey conviene que a lo menos mida de la ciudad auténtica la torre. La ley existe, ¿̇más por quién cumplida? Por nadie, el pastor que marcha al frente rumiar puede, más su uña no está hendida; 
y puesto que a su guía ve la gente

herir la presa de ella codiciada, nada pregunta y en pacer consiente.

Bien ves que la conducta depravada

es la causa que al mundo torno inmundo, no que nuestra natura esté añada.

Solía Roma, por quien fue fecundo, con un sol señalarnos el camino de Dios, y con el otro aquel del mundo. Apagó el uno al otro, y su destino unen [báculo] y espada; [...] si la mano ${ }^{4}$ se dan por fuerza, es puro desatino porque, juntos, ninguno es soberano. ${ }^{5}$ (Purgatorio, XVI, 88-112)

\footnotetext{
${ }^{4}$ En la versión de Crepo dice "tiara", pero el texto toscano dice "pasturale", que Ruiz traduce como "báculo" y me parece que es más apropiado. La omisión del " $y$ " se da por motivos métricos.

${ }^{5}$ Las citas de la Divina comedia provienen de Crespo (1981) y se contrastan en las notas con el texto toscano proveniente de González Ruiz (1994). Las citas del Monarquía provienen de Gutiérrez (1994).
}

\author{
I'anima semplicetta che sa nulla \\ salvo che, mossa di lieto fattore, \\ volontier torna a ció che la trastulla, \\ Di picciol bene in pria sente sapore; \\ quivi s'inganna, e dietro ad esso corre \\ se guida o fren non torce sou amore. \\ Onde convenne legge per fren porre; \\ convenne rege aver, che discernesse \\ de la vera cittade almen la torre \\ Le leggi son, ma chi pon mano ad esse? \\ Nullo: però che'l pastor che procede, \\ rumiar può, ma non ha l'hungie fesse; \\ per che la gente, che sua guida vede \\ pur a quel ben fidire ond'ella è ghiotta \\ di quel ben qui pasce, a più oltre non chiede.
}


Por una parte, Dante reconoce que el pecado es algo propio del pecador. El alma tiende a las cosas que le generan placer, lo cual es natural y no un vicio per se. En el canto XVIII, Virgilio explica que parte de la libertad consiste en dar freno al deseo porque podemos distinguir entre lo que es bueno y malo, aunque relega una explicación más detallada a su encuentro con Beatriz en el paraíso, ya que éste es un asunto de la fe (González, 1994, 316). Por otra parte, encontramos que uno de los motivos por los cuales Dante quiere separar el poder espiritual del poder temporal es por la guía espiritual que éste representa. Si bien existen las leyes orientadas a cuidar el buen comportamiento, Dante ve que las autoridades eclesiásticas de su tiempo las conocen, aunque no las cumplen; metafóricamente, rumean, pero no tienen la pezuña hundida. ${ }^{6}$ El papa al fallar como ejemplo, hace que su rebaño, la gente, también se desvíe de su camino. Dante pone inmediatamente una contraposición al referir a los dos soles de Roma, o sus dos poderes. Mientras uno se encargaba de proveer las condiciones de la paz para el óptimo medio de la felicidad del hombre, el otro poder se encargaba de encaminar al hombre a la recta vía que Dios manda. Esto es algo que Dante consideraba perdido, pues, al estar unidos, ninguno de los dos cumple su fin. A modo de ejemplo, refiere a las contiendas realizadas por Federico II en las regiones del Río Po. Finalmente, Dante concluye su diálogo con Marco indicando que ahora entiende que a los levitas les fue quitada su herencia para centrarse únicamente en lo divino. El suceso referido lo encontramos en el libro de los Hechos y versa del siguiente modo:

Ben puoi veder che la mala condotta

è la cagion che'l mondo ha fatto reo,

e non la natura che'n voi sia corrotta.

Soleva Roma, che'l buon mondo feo,

duo soli aver, che l'una e l'altra strada

facean vedere, e del mondo e di Deo.

L'un l'altro ha spento; ed e guinta la spada

col pasturale, e l'un con l'altro insieme

per viva forza mal conven che vada

pero chè, giunti, l'un l'altro non teme.

${ }^{6}$ Un comentario extendido de esta metáfora se halla en (Barceló, 2003, pp. 325 - 327). 
El señor le dijo a Aarón: - Tú no recibirás herencia en su tierra, ni tendrás una parte entre ellos. Yo mismo seré tu herencia y tu parte en medio de los hijos de Israel. Yo doy como herencia a los hijos de Leví todos los diezmos de Israel en compensación por los servicios que prestan en la tienda del encuentro. De esta manera, los israelitas no tendrán necesidad de acercarse a la tienda del encuentro, y no se harán reos del pecado ni morirán. (XVIII: 20-23, versión Reina-Valera).

Después de este pasaje tenemos que esperar hasta el canto XIX para reencontrarnos con una crítica directa el poder espiritual. Al entrar en la quinta cornisa del purgatorio, la de los avaros, Dante tiene una visión en la que se le aparece de frente una mujer deforme, aunque entre más la mira más bella aparece ésta. En palabras de Dante: tomaba "su semblante desvaído el tinte que el amor quiere" $(\mathrm{XIX}, 14 \mathrm{~s}) .{ }^{7}$ La fantasía termina cuando una mujer santa le quita sus prendas a la otra y ésta pierde su encanto. Entre comentaristas es casi unánime la interpretación: La mujer que se transforma a la vista representa a los bienes materiales, ya que estos ganan atractivo entre mayor sea la atención recibida, y la mujer santa que la desviste representa a la prudencia. ${ }^{8}$ Esto trae a cuenta lo que veíamos hace poco: el alma se place en los bienes temporales y puede perderse en ellos si no cuida de su libertad.

Igual que en el infierno, en el purgatorio la ironía es la que rige la naturaleza de los castigos. Por fijarse sólo en los bienes terrenales, los avaros mantienen su cara hacia abajo mientras Iloran para purificarse con cada lágrima. Aquí podemos notar un par de cosas.

En primer lugar, la relevancia que Dante le otorga a la eternidad, orden y unidad del cielo, pues Virgilio la reclama “¿Qué tienes que a la tierra vas mirando?” (Purgatorio, XIX, 52).9 Un reclamo similar ya había sucedido igual en el canto XIV (148-151):

\footnotetext{
${ }^{7}$ Com'amor voul, così le colorava.

${ }^{8}$ Algunos, p. ej., discuten si es la mujer santa o Virgilio, guiado por la mujer santa, quien desviste a la otra mujer (De Montalbán, 2001, 183).

9 Che hai che pur inver la terra guati?
} 
Os llama el cielo y en redor os gira

Para mostraros su belleza eterna

y el ojo vuestro hacia la tierra mira:

y os castiga quien todo lo gobierna. ${ }^{10}$

En Monarquía (I, 9), Dante sugería que debe haber un gobierno único en el mundo por ser único y ordenado el cielo; y, aún más, nos ofrece esta reflexión a partir de un pasaje de la Consolación de la filosofía (II, 8) de Boecio que versa: "Oh feliz género humano si rigiera vuestras almas el amor que rige el cielo", ${ }^{11}$ el cual parece un candidato plausible para la inspiración de Dante de su último verso en la Comedia, en el que afirma que su voluntad era movida por el amor que mueve al Sol y a las demás estrellas.

En segundo lugar, también nos damos cuenta de que las lágrimas y, por tanto, el dolor, es algo explícitamente concomitante a este castigo. Si bien en otras partes del purgatorio nos encontramos a personajes que Iloran, en ninguna otra cornisa se establece esta relación necesaria entre la condena y las lágrimas. Como lo hace explícito Adriano V, ésta es la pena más amarga de todo el purgatorio (Purgatorio, XIX, 117).

Prosiguiendo con caracterización de este último personaje, Adriano $\mathrm{V}$ fue un papa cuya duración en el poder espiritual fue muy corta, apenas 37 días; sin embargo, Dante plantea que ese fue tiempo suficiente para que él se diera cuenta de cuán difícil es llevar el sagrado manto sin mancharlo, como dando a entender que todo aquel que ocupe ese puesto se coloca en una posición propensa a la avaricia. Lo más triste de esta situación es que por la avaricia el mismo Adriano $\mathrm{V}$ dice que todas las acciones se volvieron vanas. La escena concluye de la siguiente forma: Dante,

10

Chiamavi 'I cielo e 'ntorno vi si gira, mostrandovi le sue bellezze etrerne, e l'occhio vostro pur a terra mira; onde vi batte chi tutto discerne

${ }^{11}$ Reproduzco aquí el verso de Boecio como aparece en (Gutiérrez, 1994, p. 703). 
queriendo guardar respeto por la figura de Adriano $V$, intenta arrodillarse frente a él, a lo cual éste le recuerda que no hay matrimonio después de la muerte, por lo cual el respeto que se le debe como jefe de la Iglesia ya no es algo que le corresponda. De esta forma, Adriano $V$ se nos muestra como un hombre que, al fin y al cabo, sólo es un hombre como todos los demás y que debe enfrentar solo el juicio de Dios. Esto podría ser una oposición a la escena del infierno en la que Dante responde al papa simoniaco Nicolás III y cuyo discurso no toma un tono más elevado por el respeto que Dante le tiene a las llaves:

Y, pues la reverencia me lo veda,

ya que tuviste las supremas llaves,

cuando estabas allá, en la vida leda,

no he de decir palabras aún más graves.

Oh avaricia en el que el mundo se contrista:

hundir al bueno, alzar al malo sabes. ${ }^{12}$ (Infierno, XIX, 100-105)

Para concluir con nuestra revisión del purgatorio, analicemos el canto XX. Nuestro personaje central se refiere a sí mismo como Hugo Capeto, aunque los comentaristas coinciden en que por su descripción en realidad se trata de Hugo el grande, padre de Hugo Capeto (González, 1994, p. 291;

De Montalbán, 2001, 187). En todo caso, habla quien fuera:

raíz de la malvada planta

que da a la cristiandad sombra agobiante

por la que buenas mieses no levanta. ${ }^{13}$ (Purgatorio, XX, 43-45)

12

E se non fosse ch'anchor lo mi vieta

la reverenza delle Somme chiavi

che tu tenesti nella vita lieta,

io userei parole ancor più gravi ;

che la vostra avarizia il mondo attrista,

calcando i buoni e sollevando i pravi 
Específicamente se refiere a los reyes de la dinastía Capeto, la cual duró de 987 a 1328 por línea directa (Echeverría, 2013, p. 329), lo cual indica una crítica abierta a la monarquía francesa en los tiempos de Dante.

En este canto, por vía de Hugo el grande, Dante reprocha los males de ciertos reyes franceses. Refiere a las usurpaciones de Felipe "El hermoso" en "Pontieu, Gascuña" y Felipe Augusto en "Normandía" (Purgatorio, XX, 66); a Carlos de Anjou, quien hizo decapitar a Conradino, el último rey de la dinastía Hohenstaufen y heredero al reino de Nápoles, cuando éste tenía 16 años, y es este mismo Carlos quien se creía que era el supuesto asesino de Santo Tomás de Aquino, ya que éste podía oponérsele en el concilio de Lión (Crespo, 1981, 281; Echevería, 2013, p. 330). Más adelante en el mismo canto (vv. 79-81) también se refiere a él como el que regateó a su hija haciendo alusión al matrimonio que pactó entre esta y el marqués de Ferrara Azzo VII (vv.79-81). Particularmente, dice de uno:

Veo un tiempo, que ya se está acercando, en el que Francia nuevo Carlos lanza para irse, con los suyos, ostentando.

Sale sin armas, sólo con la lanza con que Judas justara, y con su punta hacia Florencia reventar la panza. ${ }^{14}$ (Purgatorio, XX, $70-75$ )

Io fui radice de la mala pianta che la terra cristiana tutta aduggia, si che boun fruto rado se ne schianta

Tempo vegg'io, non molto dopo ancoi che tragge un altro Carlo fuor di Francia per far conocer meglio e sè e' suoi Sanz' arme n' esce e solo con la lancia con la qual giostrò Guida, e quella ponta sì ch'a Fiorenza fa scoppiar la pancia 
Aquí muestra Dante uno de los episodios más personales. Se refiere a Carlos de Valois, quién bajó a Florencia como presunto pacificador mandado por Bonifacio VIII; sin embargo, lo que en realidad sucedió fue una neutralización de la facción blanca de los güelfos para establecer la primacía de la facción negra y con ello el poder del papa en Florencia. Dante estaba en una misión diplomática en Roma, pero este fue el hecho que marcó su destierro. Aún con todo, Dante también condena que Felipe IV, "El hermoso", cautivara al vicario de Cristo haciendo alusión a la prisión de Bonifacio VIII y a su eventual muerte en la cárcel (Crespo, 1981, 282). Más allá de la enemistad personal, Dante seguía reconociendo la figura del sucesor de Pedro. Finalmente, dice sobre el mismo:

\author{
Nuevo pilatos tan cruel contemplo \\ que no le sacia, y lleva sin decreto \\ las ambiciosas velas contra el templo. ${ }^{15}$ (Purgatorio, $X X, 91-93$ )
}

El hecho referido es el ataque a los jefes de los templarios, a los cuales quemó. Esto marcaría el fin de la orden (Crespo, 1981, 282).

En contraposición a estas acciones, Dante pone en los cantos de los avaros casos ejemplares de una vida sin avaricia. Refiere a María quien tuvo al rey de los cielos en un hospicio pobre; y también a Fabricio, un romano quien se negó las riquezas con tal de mantener su virtud. Un caso curioso es la alusión a la historia de San Nicolás. Un hombre sin dinero estaba por prostituir a sus hijas; y Nicolás, al saber de esto, donó unas monedas de oro en la noche y en secreto para que las hijas del hombre pudieran dedicarse a la vida honesta (Crespo, 1981, p. 280). El nombre posteriormente se deformó de Nicolás a Klaus (Echeverría, 2013, p. 328). En los Estados Unidos se representa esta escena con los calcetines en la chimenea. De una misma forma, también refiere 
algunos casos en los que la avaricia fue contraproducente. Los avaros recuerdan a Midas, el rey quien pidió que todo cuanto tocase fuera convertido en oro, lo cual trajo la muerte de una de sus hijas, y cuya historia, dice Dante, "toda burla viene a cuento" (Purgatorio, XX, p. 108). ${ }^{16}$ Gritan entre todos “Oh, Craso [...] si, pues lo sabes, ¿cómo sabe el oro?" (p. 116s), ${ }^{17}$ refiriendo a la muerte de Licinio Craso, quien, a su muerte, se le hizo tragar oro fundido por su insaciable sed de riquezas (Crespo, 1981, 283). Todas estas historias las cantan los avaros, aunque en la cercanía a Dante sólo Capeto las canta con fuerza mientras que los demás apenas y murmuran porque grande es la culpa de él a comparación de la de otros (Purgatorio, XX, 118-123).

Lo que hemos revisado en este trabajo es una caracterización general de la avaricia en el purgatorio en relación con el Monarquía de Dante. En su momento, sus ideas fueron revolucionarias, o cuando menos contravenían un orden establecido. Muestra de ello es la condena que sufrió este documento en 1329 por el cardenal Bertran de Pogeto, quien intentó quemarlo (González, 1994, 697). En 1559 se le incluyó en el índice de libros prohibidos por el vaticano, aunque León XIII lo sacó después por no encontrar nada en él que contradijera la doctrina cristiana. Inclusive llegó a escribir un par de bulas, Inmortale Dei y Sapientiae Cristianiae, en las que recupera algunas ideas de la división de poderes temporal y espiritual (Robles y Frayle, 1992, 20-22).

Ciertamente muchos de los argumentos que expuso Dante no pueden ser recuperados en nuestros tiempos por diversos motivos. Es opinión de Étienne Gilson que el Monarquía fue sacado del índice cuando éste ya no representaba ningún peligro porque ningún Estado moderno lo tomaría como base de su pensamiento (p. 20-22). En todo caso, considero que a fin de que no se pierdan estas reflexiones, es fundamental cuestionar las acciones de toda autoridad eclesiástica con respecto a los bienes temporales, pero esto es una tarea que se deja a cada quién para su propio tiempo y entorno.

\footnotetext{
16 per la qual cempre conven che si rida.

${ }^{17}$ Crasso / dilci, che'l sai: di che sapore è l'oro.
} 


\section{Referencias}

Barceló, J. (2003) Para leer la Divina Comedia. Biblioteca americana.

Corbett, G. (2014) The Christian Ethics of Dante's Purgatory. Medium Aevum, 83, pp. 266 - 287.

Chiclana, A. (2002) Divina comedia. Espasa Calpe.

Crespo, A. (1981) Comedia. Dos tomos. Origen.

Crespo, A. (1999) Dante y su obra. Acantilado.

Díaz, D. (2013) Una propuesta medieval de separación Iglesia-Estado: el tratado Monarchia de Dante Alighieri. En M. Ordoñez Aguilar (coord.) Ensayos de historiografía medieval, pp. 303319. Universidad Nacional Autónoma de México.

De Montalbán, E. (2001) La divina comedia. Juventud.

Echeverría, A. (2013) Divina comedia. Alianza.

González, N. (1994) La divina comedia. Texto bilingüe. En N. González Ruiz (ed.) Obras completas de Dante Alighieri, pp. 17-534, Biblioteca de Autores Cristianos.

Gutiérrez, J. L. (1994) Monarquía. En N. González Ruiz (ed.) Obras completas de Dante Alighieri, pp. 694-742, Biblioteca de Autores Cristianos.

Robles, L., y Frayle, L. (1992) Monarquía. Tecnos. 